PROCEEDINGS OF THE

AMERICAN MATHEMATICAL SOCIETY

Volume 138, Number 1, January 2010, Pages 153-163

S 0002-9939(09)10053-9

Article electronically published on August 12, 2009

\title{
ON THE SOLVABILITY \\ OF THE CHARACTERISTIC DIRICHLET PROBLEM FOR LINEAR DEGENERATE PARABOLIC EQUATIONS
}

\author{
NICOLAI KUTEV, ALESSANDRO OLIARO, AND PETAR POPIVANOV
}

(Communicated by Matthew J. Gursky)

\begin{abstract}
We investigate the classical solvability for some classes of linear, degenerate equations in divergence form with prescribed Dirichlet data. Since the boundary value problem is characteristic according to Fichera on a part of the boundary, some typical nonlinear phenomena at these points are observed as boundary gradient blowups of the classical solutions in space directions. The regularity results explain the lack of hypoellipticity for special right-hand sides or boundary data for linear degenerate parabolic equations.
\end{abstract}

\section{INTRODUCTION}

In this paper we investigate the classical solvability for linear degenerate parabolic equations in divergence form

$$
L u=u_{t}-\left(a^{i j}(x, t) u_{x_{i}}\right)_{x_{j}}+b^{i}(x, t) u_{x_{i}}=0 \text { in } Q=\Omega \times\left(-t_{1}, t_{2}\right),
$$

$u(x, t)=\varphi(x, t)$ on the parabolic boundary $\gamma=\left(\partial \Omega \times\left[-t_{1}, t_{2}\right]\right) \cup\left(\Omega \times\left\{t=-t_{1}\right\}\right)$,

where $\Omega$ is a bounded $C^{\infty}$ smooth domain in $\mathbf{R}^{n}, t_{1}, t_{2}=$ const $>0$ and the summation convention under repeated indices is understood. A model example of (1.1) is the equation

$$
\begin{gathered}
L_{1} u=u_{t}-t^{k} u_{x x}+a_{1} t^{m} u_{x}=0 \text { in } Q_{1}=(-l, l) \times\left(-t_{1}, t_{2}\right), \\
u=\varphi_{1}(x, t) \text { on the parabolic boundary of } Q_{1},
\end{gathered}
$$

where $a_{1}=$ const $\neq 0$,

$$
k, m \in \mathbf{N}, k \text { is even and } k<2 m+1 .
$$

In the case that $k>2 m+1$, from the local solvability results in [12, 6], it follows that equation (1.3) is not locally solvable for special right-hand sides (or equivalently for some boundary data) even in a distributional sense in $\mathcal{D}^{\prime}$.

Received by the editors September 17, 2008.

2000 Mathematics Subject Classification. Primary 35K65, 35K20, 35 B45.

Key words and phrases. Degenerate parabolic equations, characteristic Dirichlet problem, hypoellipticity, elliptic regularization, a priori estimates.

(C)2009 American Mathematical Society Reverts to public domain 28 years from publication 
Further on we suppose that

$$
\begin{gathered}
a^{i j}, b^{i}, \varphi \in C^{\infty}(\bar{Q}), a^{i j}=a^{j i} \\
a(t)|\xi|^{2} \leq a^{i j}(x, t) \xi_{i} \xi_{j} \leq \Lambda a(t)|\xi|^{2}
\end{gathered}
$$

for every $(x, t) \in Q^{\prime} \ni \bar{Q}, \xi \in \mathbf{R}^{n}$, with a nonnegative function $a(t) \in C\left[-t_{1}, t_{2}\right]$, $a(t)>0$ for $t \neq 0, a(0)=0, \Lambda=$ const $\geq 1$;

$$
a(t) \text { has a finite order zero, }
$$

i.e. $a(t) \geq a_{0}|t|^{k}$ for some positive constant $a_{0}>0$ and some $k \in \mathbf{N}$.

Our main assumption coming from the local solvability results in $\mathcal{D}^{\prime}$ of equation (1.3) in [7] describes the admissible rate of the degeneracy of the diffusion, i.e. the principal term $a^{i j}(x, t) \xi_{i} \xi_{j}$ in comparison with the reaction, i.e. the lower order term $b^{i}(x, t) \xi_{i}$ :

$$
\left|b^{i}(x, t)\right| \leq b(t)
$$

for every $(x, t) \in \bar{Q}, b(t) \in C\left[-t_{1}, t_{2}\right]$ and $b(t) \leq a^{\sigma}(t)$ for every $t \in\left[-t_{1}, t_{2}\right]$ and some $\sigma>\frac{1}{2}-\frac{1}{2 k}$. Note that for the model equation (1.3), condition (1.8) follows for $a(t)=t^{k}, b(t)=\left|a_{1}\right| t^{m}$ and $\sigma=\frac{m}{k}>\frac{1}{2}-\frac{1}{2 k}$ from (1.4).

Under the above conditions we will prove existence and uniqueness of a classical $C^{\infty}(\bar{Q} \backslash(\partial \Omega \times\{t=0\})) \cap C^{\alpha, \alpha / 2}(\bar{Q})$ solution of (1.1), (1.2) for some $\alpha \in(0,1)$. Example 4.1 in section 4 illustrates for the model equation (1.3) that this result is sharp by constructing an explicit solution of the boundary value problem.

Linear degenerate elliptic and parabolic equations appear in simple models of real diffusion, filtration processes, and Brownian motion (see, for example, [1], 13, [8], 9]). They were investigated with different methods, for example, with energy methods by Kohn and Nirenberg [ $[8]$ or with regularization of the equation and the Bernstein technique of a priori estimates by Oleinik and Radkevich [13. According to the pioneering papers of Fichera [3, 4, some part of the boundary should be free from boundary data if the equation is a degenerate one. More precisely, for general linear second order degenerate elliptic equations

$$
\begin{array}{r}
\sum_{i, j=1}^{n} a^{i j} u_{x_{i} x_{j}}+\sum_{i=1}^{n} b^{j}(x) u_{x_{i}}+c(x) u=f(x) \text { in } \Omega, \\
\sum a^{i j}(x) \xi^{i} \xi^{j} \geq 0 \text { for every } \xi \in \mathbf{R}^{n},
\end{array}
$$

Fichera introduced the function

$$
\beta(x)=\sum_{i=1}^{n}\left(b^{i}(x)-\sum_{j=1}^{n} a_{x_{j}}^{i j}(x)\right) \nu^{i},
$$

where $\nu(x)$ is the outer unit normal to $\partial \Omega$. Then the Dirichlet data should be prescribed on the parts of the boundary $\partial \Omega$ where $\sum a^{i j}(x) \nu^{i} \nu^{j}>0$ or $\sum a^{i j}(x) \nu^{i} \nu^{j}=$ 0 but $\beta>0$. The sign of the Fichera function $\beta(x)$ is invariant under every smooth nondegenerate change of the independent variables. What does happen with the solution when the data are prescribed on the rest of the boundary, where the Dirichlet problem is characteristic? The best explanation of the phenomena which appear is given from the theory of viscosity solutions developed by M. Crandall and P.-L. Lions (for more details see 2], 5]). If, according to Fichera, the characteristic part of the boundary is with nonzero $(n-1)$-dimensional measure, then this part of the 
topological boundary de facto is an "interior domain" for equation (1.9). More precisely, from the definition of a weak viscosity Dirichlet problem (see Definition 7.4 in [2]) the solution detaches from the data. For example, the same behavior of the solution can be observed by the solutions of the heat equation on the upper base of the cylinder. Note that this part of the boundary for the heat equation is a characteristic one according to the theory of Fichera.

When the characteristic set on the boundary has zero measure in $\mathbf{R}^{n}$, then it is not clear what happens with the solution. It is not realistic to expect that the solution detaches from the smooth boundary data exactly at one characteristic point on the boundary. Our conjecture is that, in general, the normal to the boundary derivative of the solution blows up at this point. There is a simple example of Krylov (see Remark 4.1 in [9]) which confirms our conjecture.

\section{Example 1.1.}

$$
\begin{array}{r}
u_{t}-\Delta u=f(x, t) \text { in } B=\left\{\sum_{i=1}^{n} x_{i}^{2}+t^{2}<1\right\}, \\
u=0 \text { on } \partial B .
\end{array}
$$

The north pole $(0,1)$ of the unit ball $B$ is a characteristic point for the Dirichlet problem (1.10) and should be free from boundary data. When $f \in C^{2}(\bar{B})$ and $n \geq 3$ the classical solution of (1.10) has bounded second derivatives in $\bar{B}$. However, for $n=1$ and $f(0,1) \neq 0$ the time derivative $\left|u_{t}(x, t)\right|$ blows up for $(x, t) \rightarrow(0,1)$.

Let us point out that equation (1.3) for $a_{1}=0$ was considered by Helffer 7 ] and Matsuzawa [11. They proved $C^{\infty}$ hypoellipticity for $L_{1}$ and therefore local solvability for the adjoint operator $L_{1}^{*}$. When $a_{1} \neq 0$, it was shown in [6] and [12. that for $k>2 m+1$ equation (1.3) is locally nonsolvable in the set of the ultradistributions, while for $k \leq 2 m+1$ the operator $L_{1}^{*}$ is locally solvable and $L_{1}$ is $C^{\infty}$ hypoelliptic.

In this paper we connect the local solvability results with the global weak and classical solvability of (1.1), (1.2). More precisely, we show that the solution is $C^{\infty}$ smooth except for the characteristic points $\partial \Omega \times\{t=0\}$, and higher regularity results at these points depend on some additional conditions on the boundary data and the rate of the degeneracy of the diffusion and the reaction terms in equation (1.1).

The paper is organized in the following way. In section 2 the basic definitions, notation and the main results are given, while section 3 deals with the proof of the main results. In section 4 the solution of the model equation (1.3) is explicitly found.

\section{BASIC DEFINITIONS AND MAIN RESUltS}

In order to formulate the results let us recall some definitions of Banach spaces in [10. $W_{2}^{1,0}(Q)$ is the Hilbert space with a scalar product

$$
(u, v)_{W_{2}^{1,0}(Q)}=\int_{Q}\left(u v+u_{x_{k}} v_{x_{k}}\right) d x d t ;
$$

$W_{2}^{1,1}(Q)$ is the Hilbert space with a scalar product

$$
(u, v)_{W_{2}^{1,1}(Q)}=\int_{Q}\left(u v+u_{x_{k}} v_{x_{k}}+u_{t} v_{t}\right) d x d t
$$


$L_{q, r}(Q), 1 \leq q \leq \infty, 1 \leq r \leq \infty$, in the Banach space of all measurable functions in $\bar{Q}$ with a finite norm

$$
\|u\|_{L_{q, r}(Q)}=\left(\int_{-t_{1}}^{t_{2}}\left(\int_{\Omega}|u(x, t)|^{q} d x\right)^{r / q} d t\right)^{1 / r}
$$

$V_{2}(Q)$ is the Banach space of all elements of $W_{2}^{1,0}(Q)$ having a finite norm

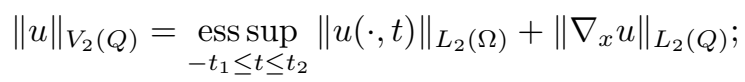

$V_{2}^{1,0}(Q)$ is the Banach space of all elements of $V_{2}(Q)$ that are continuous in $t$ in the norm of $L_{2}(\Omega)$, i.e.

$$
\|u(\cdot, t+h)-u(\cdot, t)\|_{L_{2}(\Omega)} \rightarrow 0 \text { when } h \rightarrow 0
$$

with a norm

$$
\|u\|_{V_{2}^{1,0}(Q)}=\sup _{-t_{1} \leq t \leq t_{2}}\|u(\cdot, t)\|_{L_{2}(\Omega)}+\left\|\nabla_{x} u\right\|_{L_{2}(Q)}
$$

and $C^{\alpha, \alpha / 2}(\bar{Q}), \alpha \in(0,1)$, is the Banach space of all continuous functions which are Hölder continuous with exponent $\alpha$ on $x$ and Hölder continuous with exponent $\frac{\alpha}{2}$ with respect to $t$.

A zero over the spaces means the closure of $C_{0}^{\infty}$ functions which are zero on the parabolic boundary in the corresponding norms.

We will use the standard definition of generalized solutions (see, for example, sec. 3 , ch. 3 , in [10]).

Definition 2.1. A function $u(x, t) \in V_{2}(Q)$ is a generalized solution of (1.1), (1.2) if $u(x, t)-\varphi(x, t) \in \stackrel{\circ}{V}_{2}(Q)$, and the identity

$$
\begin{aligned}
& \int_{\Omega}(u(x, \tau)-\varphi(x, \tau)) \eta(x, \tau) d x-\int_{Q_{\tau}}(u-\varphi) \eta_{t} d x d t \\
& \quad+\int_{Q_{\tau}}\left(a^{i j}(u-\varphi)_{x_{i}} \eta_{x_{j}}+b^{i}(u-\varphi)_{x_{i}} \eta\right) d x d t=-\int_{Q_{\tau}} L \varphi \cdot \eta d x d t
\end{aligned}
$$

holds for every $-t_{1} \leq \tau \leq t_{2}, Q_{\tau}=Q \cap\{t \leq \tau\}$ and for every $\eta \in \stackrel{\circ}{W^{1,1}}(Q)$.

Let us also recall the compatibility condition of the data on $\partial \Omega \times\left\{t=-t_{1}\right\}$ (see (5.6)-(5.8) in [10]). These conditions consist in the fact that the derivatives $\frac{\partial^{p} u}{\partial t^{p}}$ for $(x, t) \in \partial \Omega \times\left\{t=-t_{1}\right\}$, which can be determined for $t=-t_{1}$ by means of equation (1.1) and the initial conditions, must satisfy the boundary condition (1.2).

Theorem 2.2. Suppose (1.5)-(1.8) hold and the compatibility conditions of the data on $\partial \Omega \times\left\{t=-t_{1}\right\}$ up to infinite order are satisfied. Then problem (1.1), (1.2) has a unique classical solution $u(x, t) \in C^{\infty}(\bar{Q} \backslash\{\partial \Omega \times\{t=0\}\}) \cap C^{\alpha, \alpha / 2}(\bar{Q})$ for some $\alpha \in(0,1)$.

Remark 2.3. The result in Theorem 2.2 holds for arbitrary real positive constants $k \notin \mathbf{N}$ if the condition for $\sigma$ in $(1.8)$ is replaced with the inequality $\sigma>\frac{1}{2}-\frac{1}{2([k]+1)}$, where $[k] \in \mathbf{N},[k] \leq k$. 
Remark 2.4. The increasing regularity of the solutions at the characteristic points $\partial \Omega \times\{t=0\}$ can be proved for more general equations in nondivergence form,

$$
u_{t}-a^{i j}(x, t) u_{x_{i} x_{j}}+b^{i}(x, t) u_{x_{i}}=0 \text { in } Q, \quad u=\varphi \text { on } \gamma,
$$

under additional assumptions such as $\varphi(x, t) / a(t) \in C^{\infty}\left(\partial \Omega \times\left[-t_{1}, t_{2}\right]\right), b^{i}(x, t) / a(t)$ $\in C^{\infty}(\bar{Q})$. In this case, from the proof of Theorem 2.2, it follows that $u(x, t) \in$ $C^{\infty}(\bar{Q})$.

\section{Proof of the main Results}

The first step in the proof of Theorem 2.2 is to regularize equation (1.1); i.e. for every sufficiently small positive constant $0<\epsilon \leq \epsilon_{0}$ we consider the problem

$$
\begin{aligned}
L^{\epsilon} v^{\epsilon} & =v_{t}^{\epsilon}-\left(\left(a^{i j}(x, t)+\epsilon \delta_{i}^{j}\right) v_{x_{i}}^{\epsilon}\right)_{x_{j}}+b^{i}(x, t) v_{x_{i}}^{\epsilon}=f^{\epsilon}(x, t) \text { in } Q, \\
v^{\epsilon} & =0 \text { on } \gamma,
\end{aligned}
$$

where $\delta_{i}^{j}=1$ for $i=j, \delta_{i}^{j}=0$ for $i \neq j$, and $f^{\epsilon}(x, t)=-L^{\epsilon} \varphi$. Let

$$
\begin{aligned}
& a^{\epsilon} \in C^{\infty}\left[-t_{1}, t_{2}\right], a(t)<a^{\epsilon}(t) \leq a(t)+\epsilon \text { for every } 0<\epsilon \leq \epsilon_{0} \text { and } \\
& \left\|a^{\epsilon}-a\right\|_{C\left[-t_{1}, t_{2}\right]} \rightarrow 0 \text { when } \epsilon \rightarrow 0 .
\end{aligned}
$$

We change the variable $t$ :

$$
s=h^{\epsilon}(t)=\int_{0}^{t} a^{\epsilon}(\tau) d \tau, \quad h^{\epsilon} \in C^{\infty}\left[-t_{1}, t_{2}\right] .
$$

Since $h^{\epsilon}(t)$ is a strictly monotone function $h^{\epsilon}:\left[-t_{1}, t_{2}\right] \rightarrow\left[-T_{1}, T_{2}\right]$, the inverse function $\left(h^{\epsilon}\right)^{-1}(s)$ is well defined in $\left[-T_{1}, T_{2}\right]$. For convenience, further on we will omit the index $\epsilon$.

Let us consider the problem

$$
\begin{gathered}
P v=v_{s}-\left(A^{i j}(x, s) v_{x_{i}}\right)_{x_{j}}+B^{i}(x, s) v_{x_{i}}=F(x, s) \text { in } G=\Omega \times\left[-T_{1}, T_{2}\right], \\
v(x, s)=0 \text { on } \Gamma=\left(\partial \Omega \times\left[-T_{1}, T_{2}\right]\right) \cup\left\{\Omega \times\left\{s=-T_{1}\right\}\right\},
\end{gathered}
$$

where

$$
\begin{aligned}
A^{i j}(x, s) & =\left(a^{i j}\left(x, h^{-1}(s)\right)+\epsilon \delta_{i}^{j}\right) / A(s), \quad B^{i}(x, s)=b^{i}\left(x, h^{-1}(s)\right) / A(s), \\
A(s) & =a^{\epsilon}\left(h^{-1}(s)\right), \quad B(s)=b\left(h^{-1}(s)\right), \quad \phi(x, s)=\varphi\left(x, h^{-1}(s)\right), \\
F(x, s) & =f\left(x, h^{-1}(s)\right) / A(s)=-P \phi(x, s) .
\end{aligned}
$$

From (1.5)-(1.8), (3.2), (3.6), easy calculations give us

$$
A^{i j} \in C^{\infty}(\bar{G}), \quad A^{i j}=A^{j i}
$$

$$
|\xi|^{2} \leq A^{i j}(x, s) \xi_{i} \xi_{j} \leq 2 \Lambda|\xi|^{2} \text { for every }(x, s) \in \bar{G}, \xi \in \mathbf{R}^{n} \text { and } 0<\epsilon \leq \epsilon_{0} ;
$$

$$
\left|B^{i}(x, s)\right| \leq B(s) / A(s) \text { for every }(x, s) \in \bar{G} \text {. }
$$

Our second step is to apply to problems (3.4), (3.5) Theorems 4.1 and 4.2 in chapter 3 in [10] in order to prove existence of a generalized solution $v \in \stackrel{\circ}{V}_{2}^{1,0}(G)$. Then from Theorem 7.1 in chapter 3 in [10] we show that every solution $v \in \stackrel{\circ}{V}_{2}^{1,0}(G)$ satisfies the estimate

$$
\underset{\bar{G}}{\operatorname{ess} \sup }|v(x, t)| \leq C_{1}
$$


with a constant $C_{1}$ independent of $\epsilon$ and finally from Theorem 10.1 in chapter 3 in [10] that

$$
\|v\|_{C^{\alpha, \alpha / 2}(\bar{G})} \leq C_{2}
$$

for some $\alpha \in(0,1)$, with constants $\alpha, C_{2}$ independent of $\epsilon$.

For this purpose it is enough to check that conditions (1.2), (7.1) and (7.2) in chapter 3 in [10 are satisfied, because (1.3)-(1.6) follow from (7.1), (7.2). Assumption (1.2) is exactly (3.8) with $\nu=1, \mu_{1}=2 \Lambda$. As for (7.1), (7.2), for $q=\infty, 1<r<\min (1+1 / k, 1+(2 \sigma-1+1 / k) /(1+1 / k)), \kappa_{1}=(r-1) / r$ and $\mu_{1}=\left(C_{3}+C_{4}\right)^{1 / r}\left(C_{3}\right.$ and $C_{4}$ are defined below), we have from (1.8), (3.2), (3.9) the estimates

$$
\begin{aligned}
\left\|\left(B^{i}\right)^{2}\right\|_{L_{\infty, r}(G)}^{r}=\int_{-T_{1}}^{T_{2}} \sup _{\bar{\Omega}}\left|B^{i}(x, s)\right|^{2 r} d s & \leq \int_{-t_{1}}^{t_{2}} b^{2 r}(t) a^{1-2 r}(t) d t \\
& \leq \int_{-t_{1}}^{t_{2}} a^{2 r(\sigma-1)+1}(t) d t \leq C_{3}
\end{aligned}
$$

and

$$
\begin{aligned}
& C_{3}=\left(t_{1}+t_{2}\right)\left(\max _{\left[-t_{1}, t_{2}\right]} a(t)\right)^{2 r(\sigma-1)+1}<\infty \text { when } 2 r(\sigma-1)+1 \geq 0, \\
& C_{3}=a_{0}^{2 r(\sigma-1)+1} \int_{-t_{1}}^{t_{2}}|t|^{2 r k(\sigma-1)+k} d t<\infty \text { when } 2 r(\sigma-1)+1<0
\end{aligned}
$$

because $2 r k(\sigma-1)+k>-1$;

$$
\begin{aligned}
\|F\|_{L_{\infty, r}(G)}^{r}= & \int_{-T_{1}}^{T_{2}} \sup _{\bar{\Omega}}|F(x, s)|^{r} d s=\int_{-t_{1}}^{t_{2}} \sup _{\bar{\Omega}}\left|L^{\epsilon} \varphi(x, t)\right|^{r}\left(a^{\epsilon}(t)\right)^{1-r} d t \\
\leq & \left\{2 n\left[1+\left\|a^{i j}\right\|_{L_{\infty}(\bar{Q})}+\left\|\nabla_{x} a^{i j}\right\|_{L_{\infty}(\bar{Q})}+\left\|b^{i}\right\|_{L_{\infty}(\bar{Q})}\right]\|\varphi\|_{C^{2,1}(\bar{Q})}\right\}^{r} \\
& \cdot a_{0}^{1-r} \int_{-t_{1}}^{t_{2}} t^{(1-r) k} d t=C_{4}<\infty .
\end{aligned}
$$

Here the constants $C_{3}, C_{4}$ are independent of $\epsilon$. Let us check that $u^{\epsilon}(x, t)=$ $\varphi(x, t)+v^{\epsilon}\left(x, h^{\epsilon}(t)\right) \in V_{2}^{1,0}(Q) \cap C^{\alpha, \alpha / 2}(\bar{Q})$ is a generalized solution of (1.1), (1.2). If $\eta(x, t) \in \stackrel{\circ}{W}_{2}^{1,1}(Q)$, then $\zeta(x, s)=\eta\left(x,\left(h^{\epsilon}\right)^{-1}(s)\right) \in \stackrel{\circ}{W_{2}^{1,1}}(G)$, and hence for every $-T_{1}<R \leq T_{2}$ we have from Definition 2.1 the identity

$$
\begin{aligned}
& \int_{\Omega} v^{\epsilon}(x, R) \zeta(x, R) d x-\int_{G_{R}} v^{\epsilon}(x, s) \zeta_{s}(x, s) d x d s \\
& \quad+\int_{G_{R}}\left(A^{i j}(x, s) v_{x_{i}}^{\epsilon} \zeta_{x_{j}}+B^{i}(x, s) v_{x_{i}}^{\epsilon} \zeta\right) d x d s=\int_{G_{R}} F^{\epsilon}(x, s) \zeta(x, s) d x d s,
\end{aligned}
$$

$G_{R}=G \cap\{s \leq R\}$. 
After the change of variable (3.3) we obtain for every $-t_{1}<r \leq t_{2}, r=\left(h^{\epsilon}\right)^{-1}(R)$ the equality

$$
\begin{aligned}
& \int_{\Omega}\left(u^{\epsilon}(x, r)-\varphi(x, r)\right) \eta(x, r) d x-\int_{Q_{r}}\left(u^{\epsilon}-\varphi\right) \eta_{t} d x d t \\
& \quad+\int_{Q_{r}}\left[\left(a^{i j}(x, t)+\epsilon \delta_{i}^{j}\right)\left(u^{\epsilon}-\varphi\right)_{x_{i}} \eta_{x_{j}}+b^{i}(x, t)\left(u^{\epsilon}-\varphi\right)_{x_{i}} \eta\right] d x d t \\
& \quad=-\int_{Q_{r}} L^{\epsilon} \varphi \cdot \eta d x d t .
\end{aligned}
$$

Moreover, easy calculations give us for every fixed $\epsilon>0$ the estimates

$$
\left\|u^{\epsilon}\right\|_{V_{2}^{1,0}(Q)}<\infty, \quad \lim _{\tau \rightarrow 0}\left\|u^{\epsilon}(\cdot, t+\tau)-u^{\epsilon}(\cdot, t)\right\|_{L_{2}(\Omega)}=0 .
$$

Since $\left\|h^{\epsilon}\right\|_{C^{1}\left[-t_{1}, t_{2}\right]} \leq\left(t_{1}+t_{2}\right)\left(\max _{\left[-t_{1}, t_{2}\right]} a(t)+1\right)=C_{5}, C_{5}$ is independent of $\epsilon$, we have from (3.11) the inequality

$$
\left\|u^{\epsilon}\right\|_{C^{\alpha, \alpha / 2}(\bar{Q})} \leq\|\varphi\|_{C^{\alpha, \alpha / 2}(\bar{Q})}+C_{2} C_{5}=C_{6},
$$

where $C_{6}$ is independent of $\epsilon$.

Let $\eta \in C_{0}^{\infty}(Q)$ and $r=t_{2}$ in (3.12) so that after integration by parts the identity

$$
\int_{Q} u^{\epsilon} \eta_{t} d x d t-\int_{Q} u^{\epsilon}\left[\left(\left(a^{i j}(x, t)+\epsilon \delta_{i}^{j}\right) \eta_{x_{j}}\right)_{x_{i}}+\left(b^{i}(x, t) \eta\right)_{x_{i}}\right] d x d t=0
$$

holds.

From (3.13), the Arzelà-Ascoli Theorem and the Cantor diagonalization process, there exists a subsequence $\epsilon_{N} \rightarrow 0$ such that $u^{\epsilon_{N}}(x, t) \rightarrow u(x, t) \in C^{\alpha, \alpha / 2}(\bar{Q})$ when $\epsilon_{N} \rightarrow 0$. After the limit $\epsilon_{N} \rightarrow 0$ in (3.14) we obtain that $u(x, t)$ satisfies the same equality with $\epsilon=0$; i.e., $u(x, t)$ is a generalized solution of (1.1) in $\mathcal{D}^{\prime}(Q)$.

The third step in the proof is to apply the hypoellipticity results in [7, [11, [13].

Theorem 1.1 (Helffer [7). Suppose that the operator

$$
L_{2}=\frac{\partial}{\partial t}-A_{2 m}\left(x, t, \frac{1}{i} \frac{\partial}{\partial x_{k}}\right)+\sum_{j=0}^{2 m-1} A_{j}\left(x, t, \frac{1}{i} \frac{\partial}{\partial x_{k}}\right) \text { in } Q
$$

with $C^{\infty}(\bar{Q})$ coefficients $A_{j}(x, t, \xi)$ which are homogeneous polynomials of order $j$ with respect to $\xi \in \mathbf{R}^{n}$ satisfies the following assumptions:

(H1)

$$
\Re\left(\int_{-t^{\prime}}^{t} A_{2 m}(x, s, \xi) d s\right) \geq c\left|t-t^{\prime}\right|^{k+1}|\xi|^{2 m}
$$

for every $\left(x, t, t^{\prime}, \xi\right) \in \Omega \times\left(-t_{1}, t_{2}\right)^{2} \times \mathbf{R}^{n}, t>t^{\prime}$;

(H2) there exist constants $\theta, \tau$ such that for every $0 \leq j \leq 2 m$ and for every $(\alpha, \beta) \in \mathbf{N}^{n} \times \mathbf{N}^{n}$ satisfying

$$
|\alpha|+|\beta|+j>0, \quad(|\alpha|+j) \theta+(|\beta|+j) \tau \leq 1
$$

the estimates

$$
\begin{aligned}
& \quad\left|\frac{\partial^{\alpha+\beta}}{\partial x^{\beta} \partial \xi^{\alpha}} A_{2 m-j}(x, t, \xi)\right| \\
& \quad \leq C_{\alpha \beta j}\left|\Re A_{2 m}\right|^{1-(|\alpha|+j) \theta-(|\beta|+j) \tau}|\xi|^{2 m[(|\alpha|+j) \theta+(|\beta|+j) \tau]-|\alpha|-j}
\end{aligned}
$$

hold for every $(x, t, \xi) \in Q \times \mathbf{R}^{n}$ and for some constants $C_{\alpha \beta j}$; 
(H3) $\theta$ and $\tau$ satisfy the inequality

$$
2 m(\tau+\theta) \frac{k}{k+1}<1 .
$$

If $u \in \mathcal{D}^{\prime}(Q)$ is a generalized solution of $L_{2} u=0$, then $u \in C^{\infty}(Q)$.

Assumptions (H1)-(H3) in Theorem 1.1 in [7] hold for operator $L$ with $m=1$, $k \in \mathbf{N}, A_{2}=a^{i j}(x, t) \xi_{i} \xi_{j}, A_{1}=\sum_{j=1}^{n}\left(\sum_{h=1}^{n} a_{x_{h}}^{h j}(x, t)+b^{j}(x, t)\right) \xi_{j}$ and $A_{0}=0$. We have in fact

$$
\begin{aligned}
\Re\left(\int_{t^{\prime}}^{t} A_{2}(x, s, \xi) d s\right) & =\int_{t^{\prime}}^{t} a^{i j}(x, s) \xi_{i} \xi_{j} d s \\
& \geq a_{0}|\xi|^{2} \int_{t^{\prime}}^{t}|s|^{k} d s \geq \frac{a_{0}}{k+1}|\xi|^{2}\left|t-t^{\prime}\right|^{k+1} .
\end{aligned}
$$

As for conditions (H2), (H3) we choose $\theta=0, \tau=\frac{1}{2}+\frac{1}{2 k}-\epsilon_{1}$ for some sufficiently small $\epsilon_{1}>0$. For all indices $j, \alpha, \beta$ satisfying the inequalities

$2 k\left(\frac{1}{2}+\frac{1}{2 k}-\epsilon_{1}\right) /(k+1)=1-\frac{2 \epsilon_{1} k}{k+1}<1,|\alpha|+|\beta|+j>0, \quad(|\beta|+j)\left(\frac{1}{2}+\frac{1}{2 k}-\epsilon_{1}\right) \leq 1$, one has to check the estimates in (H2). Condition (3.16) is satisfied only by the indices

$$
\begin{aligned}
\text { i) } j & =1 \text { and } \alpha=\beta=0 \text { or }|\alpha|=1, \beta=0, \\
\text { ii) } j=0, \beta=0 \text { and }|\alpha|=1 \text { or }|\alpha|=2, & \\
\text { iii) } j & =0,|\beta|=1 \text { and } \alpha=0,|\alpha|=1 \text { or }|\alpha|=2 .
\end{aligned}
$$

In case (3.17) $\mathrm{i}$ the estimate in $(\mathrm{H} 2)$ is

$$
\begin{aligned}
& \left|\frac{\partial^{\alpha+\beta}}{\partial x^{\beta} \partial \xi^{\alpha}} \sum_{j}\left(\sum_{h} a_{x_{h}}^{h j}+b^{j}\right) \xi_{j}\right| \\
& \quad \leq K_{1}|\xi|^{2\left[(|\beta|+1)\left(\frac{1}{2}+\frac{1}{2 k}-\epsilon_{1}\right)\right]-|\alpha|-1}\left|a^{i j}(x, t) \xi_{i} \xi_{j}\right|^{1-(|\beta|+1)\left(\frac{1}{2}+\frac{1}{2 k}-\epsilon_{1}\right)} .
\end{aligned}
$$

Since from (1.6) and (1.8) we have the inequalities

$$
\begin{aligned}
& \left|\sum_{j, h} a_{x_{h}}^{h j}(x, t) \xi_{j}\right| \leq K_{0}\left(\sum_{i, j} a^{i j} \xi_{i} \xi_{j}\right)^{1 / 2} \leq K_{0} \Lambda|\xi| a^{1 / 2}(t) \leq K_{0} \Lambda|\xi|(a(t))^{\frac{1}{2}-\frac{1}{2 k}+\epsilon_{1}}, \\
& \left|\sum_{j} b^{j}(x, t) \xi_{j}\right| \leq n|\xi| a^{\gamma}(t) \leq n|\xi|(a(t))^{\frac{1}{2}-\frac{1}{2 k}+\epsilon_{1}}
\end{aligned}
$$

for $0<\epsilon_{1} \leq \min \left(\frac{1}{2 k}, \gamma-\frac{1}{2}+\frac{1}{2 k}\right)$, then (3.18) holds in case (3.17) $)_{\mathrm{i}}$ with $K_{1}=K_{0} \Lambda+n$. In the case that $j=0$ one has to prove the estimates

$$
\left|\frac{\partial^{\alpha+\beta}}{\partial x^{\beta} \partial \xi^{\alpha}} a^{i j}(x, t) \xi_{i} \xi_{j}\right| \leq K_{2}|\xi|^{2|\beta|\left(\frac{1}{2}+\frac{1}{2 k}-\epsilon_{1}\right)}\left|a^{i j}(x, t) \xi_{i} \xi_{j}\right|^{1-|\beta|\left(\frac{1}{2}+\frac{1}{2 k}-\epsilon_{1}\right)} .
$$

Since we have $\left|a^{h j}(x, t) \xi_{j}\right| \leq \Lambda|\xi| a(t),\left|a^{h j}(x, t)\right| \leq n^{2} \Lambda a(t),\left|a_{x_{i}}^{h j}(x, t) \xi_{h} \xi_{j}\right| \leq$ $K_{0} \Lambda|\xi|^{2}(a(t))^{\frac{1}{2}} \leq K_{0} \Lambda|\xi|^{2}(a(t))^{\frac{1}{2}-\frac{1}{2 k}+\epsilon_{1}}$, using (3.19) we obtain (3.20) in the cases (3.17) ii and (3.17) $)_{\text {iii }}$ with $K_{2}=n^{2} \Lambda+K_{0} \Lambda$. 
According to Theorem 1.1 the generalized solution $u(x, t)$ of (1.1) belongs to the class $C^{\infty}(Q) \cap C^{\alpha, \alpha / 2}(\bar{Q})$. From the standard local regularity theory for uniformly parabolic equations (see [10]) it follows that

$$
u(x, t) \in C^{\infty}(\bar{Q} \backslash(\partial \Omega \times\{t=0\})) \cap C^{\alpha, \alpha / 2}(\bar{Q}), \quad \alpha \in(0,1) .
$$

Uniqueness. Suppose $u, v \in C^{\infty}(Q) \cap C^{\alpha, \alpha / 2}(\bar{Q})$ are two classical solutions of (1.1), (1.2). Then $w=u-v$ is a classical solution of (1.1) with zero boundary data. If $w \neq \equiv 0$, without loss of generality, let us suppose that $w$ attains its positive maximum at some point $\left(x_{0}, t_{0}\right) \in \bar{Q} \backslash \gamma$. Then the function $z(x, t)=e^{-c t} w(x, t)$, for $c=$ const $>0$, satisfies the equation

$$
\begin{aligned}
& z_{t}+c z-\left(a^{i j}(x, t) z_{x_{j}}\right)_{x_{i}}+b^{i}(x, t) z_{x_{i}}=0 \text { in } Q, \\
& z=0 \text { on } \gamma
\end{aligned}
$$

and attains its positive maximum at some point $\left(x_{1}, t_{1}\right) \in \bar{Q} \backslash \gamma$. Since $z\left(x_{1}, t_{1}\right)>$ $0, z_{t}\left(x_{1}, t_{1}\right) \geq 0, \nabla_{x} z\left(x_{1}, t_{1}\right)=0$ and $a^{i j}\left(x_{1}, t_{1}\right) z_{x_{i} x_{j}}\left(x_{1}, t_{1}\right) \leq 0$ (see [14]), this contradicts equations (3.21).

\section{EXPLicit SOLUTION FOR THE MODEL EQUATION}

In this section we will give an explicit formula for the solutions of the model equation (1.3) for $k=m=p$.

\section{Example 4.1.}

$$
\begin{array}{r}
u_{t}-t^{p} u_{x x}+a t^{p} u_{x}=0 \text { in } Q=(-1,1) \times(-1,1), \\
u(-1, t)=A(t), \quad u(1, t)=B(t), \quad-1 \leq t \leq 1, \\
u(x,-1)=g(x), \quad-1 \leq x \leq 1, \\
A(-1)=g(-1), \quad B(-1)=g(1) .
\end{array}
$$

By means of the Green function, the solution of (4.1) can be explicitly written as

$$
\begin{gathered}
u(x, t)=\sqrt{\frac{p+1}{4 \pi}} \int_{0}^{2} \frac{g(y-1) \exp (-a(y-1) / 2)}{\left(t^{p+1}+1\right)^{1 / 2}} \\
\cdot \sum_{n=-\infty}^{\infty}\left[\exp \left(-\frac{(p+1)(x+1-y+4 n)^{2}}{4\left(t^{p+1}+1\right)}\right)-\exp \left(-\frac{(p+1)(x+1+y+4 n)^{2}}{4\left(t^{p+1}+1\right)}\right)\right] d y \\
+\frac{(p+1)^{3 / 2} \exp (a / 2)}{2 \sqrt{\pi}} \int_{0}^{\left(t^{p+1}+1\right) /(p+1)} \frac{A((p+1) s-1)^{1 /(p+1)} \exp \left(a^{2} s / 4\right)}{\left(t^{p+1}+1-s(p+1)\right)^{3 / 2}} \\
\cdot \sum_{n=-\infty}^{\infty}(x+1+4 n) \exp \left(-\frac{(p+1)(x+1+4 n)}{4\left(t^{p+1}+1-s(p+1)\right.}\right) d s \\
+\frac{(p+1)^{3 / 2} \exp (-a / 2)}{4 \sqrt{\pi}} \int_{0}^{\left(t^{p+1}+1\right) /(p+1)} \frac{B((p+1) s-1)^{1 /(p+1)} \exp \left(a^{2} s / 4\right)}{\left(t^{p+1}+1-s(p+1)\right)^{3 / 2}} \\
\cdot \sum_{n=-\infty}^{\infty}\left[(x-1+4 n) \exp \left(-\frac{(p+1)(x-1+4 n)^{2}}{4\left(t^{p+1}+1-s /(p+1)\right)}\right)\right. \\
\left.+(x+3+4 n) \exp \left(-\frac{(p+1)(x+3+4 n)^{2}}{4\left(t^{p+1}+1-s(p+1)\right)}\right)\right] d s .
\end{gathered}
$$


Simple computations show that $u(x, t)=u_{1}(x, t)+u_{2}(x, t)+u_{3}(x, t)$, where $u_{1} \in$ $C^{\infty}(\bar{Q}), u_{2} \in C^{\infty}(\bar{Q} \backslash\{(-1,0)\}) \cap C^{2 /(p+1)}(\bar{Q}), u_{3} \in C^{\infty}(\bar{Q} \backslash\{(1,0)\}) \cap C^{2 /(p+1)}(\bar{Q})$, assuming that the compatibility conditions up to infinite order are satisfied at the points $(-1,-1)$ and $(1,-1)$. Here

$$
\begin{gathered}
u_{2}=\frac{(p+1)^{3 / 2} \exp \left(a^{2} / 2\right)}{2 \sqrt{\pi}} \int_{0}^{\frac{t^{p+1}+1}{p+1}} \frac{\left.A((p+1) s-1)^{1 /(p+1)}\right) \exp \left(a^{2} s / 4\right)}{\left(t^{p+1}+1-s(p+1)\right)^{3 / 2}}(x+1) \\
\cdot \exp \left(-\frac{(p+1)(x+1)^{2}}{4\left(t^{p+1}+1-s(p+1)\right)}\right) d s
\end{gathered}
$$

and $u_{3}$ is defined in a similar way.

Finally, let us mention some open problems. From Example 1.1 and some other counterexamples of Krylov, it is known that the dimension of the space and the curvature of the boundary at the characteristic points play an important role for the rate of the singularities at these points. What is the precise dependence and the sharp singularities of the solution?

\section{ACKNOWLEDGEMENTS}

The first author is partially supported by Bulgarian grant VU-MI-102/2005.

\section{REFERENCES}

[1] H. W. Alt, S. Luckhaus: Quasilinear elliptic-parabolic equations, Math. Z., 183 (1983), 311341. MR706391 (85c:35059)

[2] M. Crandall, H. Ishii, P.-L. Lions: User's guide to viscosity solutions of second order partial differential equations, Bull. Amer. Math. Soc. 27, 1-67 (1992). MR1118699 (92j:35050)

[3] G. Fichera: Sulle equazioni differenziali lineari ellittico-paraboliche del secondo ordine, Atti Accad. Naz. Lincei. Mem. Cl. Sci. Fis. Mat. Nat. Sez. I (8) 5 (1956), 1-30. MR0089348 $(19: 658 \mathrm{a})$

[4] G. Fichera: On a unified theory of boundary value problems for elliptic-parabolic equations of second order, Boundary Problems in Differential Equations, University of Wisconsin Press, Madison, 1960, 97-120. MR0111931 (22:2789)

[5] Y. Giga: Surface evolution equations. A level set method, Rudolph-Lipschitz Vorlesung, March, 2002, No. 44, SFB 256, Bonn, Germany.

[6] T. Gramchev, P. Popivanov, M. Yoshino: Critical Gevrey index for hypoellipticity of parabolic equations and Newton polygons, Ann. Mat. Pura Appl., 170 (1996), 103-131. MR.1441616 (98c:35029)

[7] B. Helffer: Sur l'hypoellipticité d'une classe d'opérateurs paraboliques dégénérés, in Sur quelques équations aux derivées partielles singulières, Astérisque, 19, Soc. Math. France, Paris, 1974, 79-105. MR0364843 (51:1097)

[8] J. J. Kohn, L. Nirenberg: Degenerate elliptic-parabolic equations of second order, Comm. Pure and Appl. Math., 20 (1967), 797-872. MR0234118 (38:2437)

[9] N. Krylov: On degenerate nonlinear elliptic equations, II, Math. Sb., 121, (1983), 211-232 (in Russian). MR703325 (85f:35097)

[10] O. A. Ladyzhenskaya, V. A. Solonnikov and N. N. Ural'ceva: Linear and Quasilinear Equations of Parabolic Type, Amer. Math. Soc., Providence, 1967. MR0241822 (39:3159b)

[11] T. Matsuzawa: On some degenerate parabolic equations I, Nagoya Math. J., 51 (1973), 5777; II. Nagoya Math. J., 52 (1973), 61-84. MR0333450 (48:11775) MR0333451(48:11776)

[12] A. Oliaro, P. Popivanov: Gevrey local solvability for degenerate parabolic operators of higher order, Operator Theory, Adv. and Appl., 172 (2006), 135-151. MR2308508(2008a:35176) 
[13] O. A. Oleinik, E. Radkevich: Second order equations with nonnegative characteristic form, Itogi Nauki, Akad. Nauk SSSR Vsesojuzn. Inst. Naučn. i Tehn. Informacii, Moscow, 1971. MR0457907 (56:16111)

[14] M. H. Protter, H. F. Weinberger: Maximum Principles in Differential Equations, PrenticeHall, Englewood Cliffs, NJ, 1967. MR0219861 (36:2935)

Institute of Mathematics and Informatics, Bulgarian Academy of Sciences, Acad. G. Bontchev Street, Bl. 8, 1113 Sofia, Bulgaria

Department of Mathematics, University of Torino, Via Carlo Alberto, 10, I-10123 TORINo (TO), ITALY

E-mail address: alessandro.oliaro@unito.it

Institute of Mathematics and Informatics, Bulgarian Academy of Sciences, Acad. G. Bontchev Street, Bl. 8, 1113 Sofia, Bulgaria

E-mail address: popivano@math.bas.bg 